\title{
PARÁMETROS POBLACIONALES, FECUNDIDAD Y TASA SEXUAL DE LÍNEAS PURAS Y GENÉTICAMENTE VARIABLES DE TRICHOGRAMMA PRETIOSUM RILEY (HYMENOPTERA: TRICHOGRAMMATIDAE)
}

\author{
Adriana GUZMÁN-LARRALDE, ${ }^{1 *}$ ERNESTo CERNA-CHÁVEZ, ${ }^{1}$ EdMUNdo \\ RODRIGUEZ-CAMPOS, ${ }^{1}$ JUAN CARLOS LOYOLA-LICEA, ${ }^{2}$ MARIANO \\ FLORES DAVILA, ${ }^{1}$ OSWALDO GARCÍA-MARTÍNEZ ${ }^{1}$ \& RICHARD \\ STOUTHAMER ${ }^{2}$ \\ ${ }^{1}$ Departamento Parasitología Agrícola. Universidad Autónoma Agraria “Antonio Narro”, Calzada A. \\ Narro, Col. Buenavista, Saltillo, Coahuila, México.<adri_guzman@yahoo.com> \\ ${ }^{2}$ Instituto Tecnológico de Saltillo, Coahuila, México \\ ${ }^{3}$ Department of Entomology, University of California Riverside, CA 92521, USA.
}

Guzmán-Larralde, A., Cerna-Chávez, E., Rodriguez-Campos, E., Loyola-Licea, J. C., Flores Davila, M., García-Martínez, O. \& Stouthamer, R. 2014. Parámetros poblacionales, fecundidad y tasa sexual de líneas puras y genéticamente variables de Trichogramma pretiosum Riley (Hymenoptera: Trichogrammatidae). Acta Zoológica Mexicana (n. s.), 30(2): 378-386.

RESUMEN. Mediante la comparación de sus parámetros poblacionales, fecundidad y tasa sexual, se evaluó el desempeño de cuatro poblaciones de Trichogramma pretiosum Riley: dos líneas puras altamente endogámicas (98\%) con bajo y alto desempeño (2 y 43 respectivamente) y dos líneas genéticamente variables (producto de la combinación de 26 líneas puras) con 2 y 40 generaciones. Los resultados muestran que tanto en los parámetros poblacionales Ro, $\mathrm{T}, \lambda \mathrm{y} \mathrm{r}_{\mathrm{m}}$, como en la fecundidad, la línea genéticamente variable con dos generaciones presenta los mejores resultados seguido de la genéticamente variable con 40 generaciones, después se ubica a la línea 43 y por último a la línea pura 2 . Se compara la fecundidad y proporción sexual observada a las $48 \mathrm{~h}$ y total. La fecundidad a las $48 \mathrm{~h}$ no presenta diferencias significativas en entre las líneas genéticamente variables y la línea pura 43. La proporción sexual observada a las $48 \mathrm{~h}$, no presenta diferencias significativas entre las dos líneas genéticamente variables y la línea pura 43, relación que cambia en la proporción sexual total, donde se observó que las líneas genéticamente variables producen un mayor número de machos en los días subsecuentes.

Palabras clave: Parasitismo, parámetros poblacionales, endogamia, fecundidad, proporción sexual.

Guzmán-Larralde, A., Cerna-Chávez, E., Rodriguez-Campos, E., Loyola-Licea, J. C., Flores Davila, M., García-Martínez, O. \& Stouthamer, R. 2014. Population parameters, fecundity and sex ratio of isofemale lines and genetically variable in Trichogramma pretiosum Riley (Hymenoptera: Trichogrammatidae). Acta Zoológica Mexicana (n. s.), 30(2): 378-386.

Recibido: 19/09/2013; aceptado: 04/03/2014. 


\begin{abstract}
By comparing their population parameters, fecundity and sex ratio, we examined the performance of four genetically variable populations of Trichogramma pretiosum Riley, two highly inbred pure lines (98\%) with low and high performance ( 2 and 43 respectively) and two genetically variable lines (product of 26 inbred lines) with 2 or 40 generations. The population parameters determined were Ro, $T$, and $\lambda$ as $r_{m}$ and fertility, and the overall results indicate significant differences between all the lines. The genetically variable line with two generations had the best performance, then the genetically variable with 40 generations, then line 43 and finally the pure line 2 . Fertility and sex ratio were observed at $48 \mathrm{~h}$ and compared to overall. After 48 hours, no significant differences were found in fertility between the genetically variable lines to pure line 43 . After $48 \mathrm{~h}$, the sex ratio did not differ significantly between the two genetically variable lines and the pure line 43 . However, total sexual ratios showed higher number of males in the genetically variable lines.
\end{abstract}

Key words: Parasitism, population parameters, inbreeding, fecundity, sex ratio.

\title{
INTRODUCCIÓN
}

Trichogramma (Hymenoptera: Trichogrammatidae) es un parasitoide estricto de huevecillos, principalmente de lepidópteros (Pinto 1998). La reproducción de Trichogramma es de gran trascendencia en México y el mundo, por el número de individuos que son reproducidos y liberados periódicamente en programas de control biológico (Arredondo Bernal 2010, Pinto 1998). De los 58 centros de reproducción de organismos benéficos en todo el país, 59\% reproduce insectos y de estos centros el $91 \%$ distribuye y comercializa alguna especie de parasitoide para ser liberado en programas de control biológico, entre los que se destaca la especie de T. pretiosum Riley (Arredondo Bernal 2010). Al incrementar en condiciones de laboratorio cualquier organismo biológico, se influye en la variabilidad original de la población (Nunney et al. 2002). Esta variabilidad está ligada a todos los atributos reproductivos como fecundidad, tasa sexual, capacidad de búsqueda, tamaño, entre muchos otros.

En programas de control biológico clásico o por aumento, mantener la variabilidad genética del enemigo natural es fundamental para que pueda adaptarse al medio ambiente (Chassain \& Boulétreau 1991, Hopper et al. 1993, Wajnberg 2004). En ambos casos se necesita liberar gran cantidad de individuos, por lo que al incrementar el número de individuos en el proceso de reproducción en cautiverio afectamos su variabilidad, su capacidad, desempeño y calidad de la misma (Nunney 2003). Iniciar una población con pocos individuos puede generar un cuello de botella, al tener poca variabilidad genética que no refleja el total de alelos presentes en la población original (Hufbauer \& Roderick 2005). Otro factor en la pérdida de variabilidad puede darse debido a que algunos individuos están mejor adaptados a las condiciones artificiales que otros, promoviendo la selección y/o eliminación de algunos alelos, dando lugar a un proceso conocido como "domesticación" (Bartlett 1984, Allendorf 1986, Nunney et al. 2002), y esta eliminación de variabilidad puede conducir a un bajo desempeño en campo (Woodworth et al. 2002, Frankham 2005).

Para evitar la pérdida de variabilidad, algunos investigadores han sugerido dividir la población de campo en líneas puras y posteriormente mezclar el mayor número 
de líneas puras (con un mínimo de generaciones) antes de liberarlas, para recuperar la variabilidad original, y de esta manera, evitar la domesticación (Roush \& Hopper 1995, Kalyebi et al. 2005, Nunney 2006). Las líneas puras tienen de muy poca a ninguna variabilidad entre sus alelos, ya que son homocigotos para cada característica. Al no tener variabilidad que perder, no tienen cambios en el tiempo (excepto a través de mutaciones). Un ejemplo de su efectividad se puede observar en el uso de Encarsia formosa Gahan, 100\% homocigota por su partenogénesis telitoquia, usada con éxito en Europa para el control de la mosquita blanca Trialeurodes vaporariorum (Westwood), en invernaderos desde 1930 (van Lenteren et al. 1997). A pesar de las recomendaciones sobre el uso de líneas puras hay un número muy reducido de trabajos en parasitoides que prueben esta hipótesis (Sorati et al. 1996, Woodworth et al. 2002, Guzmán-Larralde et al. 2013) en donde se elijan y comparen líneas puras sin influencia del hospedero y poblaciones genéticamente variables con un número distinto de generaciones.

Para determinar y comparar el desempeño de líneas puras y poblaciones, y elegir aquella con mejores características reproductivas, se han utilizado distintos métodos. Uno de ellos son las tablas de vida de edad específica, que son consideradas como una herramienta para estimar parámetros de crecimiento, desarrollo y reproducción, al incluir en su análisis, sobrevivencia, tiempo y proporción sexual (Lotka 1907, Lewis 1942, Leslie 1945, Southwood 1978, Bellows Jr et al. 1992, Maia et al. 2000, Badii \& Castillo. 2009). Algunos investigadores han utilizado tablas de vida con Trichogramma lo que facilita la comparación de las características reproductivas de las poblaciones en los diferentes estudios (Pak \& Oatman 1982, Pratissoli \& Parra 2000, Haile et al. 2002, Pratissoli et al. 2004, Samara et al. 2008, Iranipour et al. 2009). Otros métodos relacionados con la evaluación de la calidad y desempeño en parasitoides son aquellos que usan cálculos de fecundidad y proporción sexual (Waage \& Ming 1984, Cerutti \& Bigler 1995, Kazmer \& Luck 1995, Sorati et al. 1996, Antolin 1999).

Por lo anterior el presente estudio tiene como objetivo comparar el efecto de la reproducción en laboratorio con el desempeño de líneas puras y genéticamente variables, para proporcionar evidencia de los beneficios de subdividir una población de campo, evitando la domesticación y manteniendo la variabilidad genética de $T$. pretiosum.

\section{MATERIAL Y MÉTODOS}

Obtención del material biológico. Las líneas puras de $T$. pretiosum fueron colectadas en 2008, en campos de tomate de la Universidad de California (estación Irvine) en Estados Unidos y desarrolladas en huevos de Manduca sexta Paphus. Cada hembra colectada de manera individual copuló con su propia progenie (hermanados) durante nueve generaciones. Las líneas puras resultantes tuvieron un coeficiente de 
consanguinidad del 87\% (Li 1955). Posteriormente, las colonias se mantuvieron indefinidamente en el orden de cientos de individuos en Ephestia kuehniella (Zeller). El experimento se realizó usando una incubadora a $25^{\circ} \mathrm{C}, 16 / 8$ horas (luz/obscuridad) a $30 \%$ de humedad relativa.

Comparación de las poblaciones. Se compararon cuatro poblaciones de manera simultánea. Para lo cual en un trabajo previo, se analizaron los parámetros reproductivos de 26 líneas puras (Guzmán-Larralde et al. 2013). Esta información permite seleccionar dos poblaciones, una con alto y otra con bajo desempeño (nombradas como línea 43 y 2 respectivamente). Las otras dos poblaciones son una mezcla "genéticamente variable" (como resultado de la combinación de las 26 líneas puras, incluyendo a la 43 y 2) con dos generaciones (GV2) y con 40 generaciones (GV40).

De cada una de las poblaciones se usaron 25 hembras a las 24 horas de su emergencia, éstas se colocaron individualmente en un tubo de cristal con aproximadamente 100 huevecillos de E. kuehniella y una pequeña gota de miel. Cada 2 días (48 h) los huevecillos se renovaron y se colocaron en viales separados hasta la muerte de la hembra. A la emergencia de la progenie se contabilizó y se determinó el sexo.

Análisis matemático y estadístico. La comparación del desempeño se hizo mediante el cálculo de los parámetros poblacionales: Tasa neta de reproducción, $(\mathrm{Ro}=$ $\sum$ lxmx), el número de hembras nacidas por cada madre, en cada generación; Tiempo generacional, $\left(\mathrm{T}=\sum \mathrm{x} \cdot \mathrm{xmx} / \sum \operatorname{lxmx}\right)$ intervalo de tiempo medio, entre el nacimiento de los individuos de una generación y la de la próxima generación; Tasa finita de incremento $\left(\lambda=\mathrm{e}^{\mathrm{rm}}\right)$ número de individuos que se agrega a la población por individuo y por tiempo; Tasa intrínseca de crecimiento, $\mathrm{r}_{\mathrm{m}},\left(\sum \mathrm{e}^{-r m^{x}} 1 \mathrm{xmx}=1\right)$ potencial de crecimiento de una población. El procedimiento para determinar la fecundidad a 48 horas, y total se llevó a cabo siguiendo la metodología descrita en el trabajo de (Guzmán-Larralde et al. 2013), y el cálculo de la proporción sexual por medio del método descrito en (Wilson \& Hardy 2002). Los datos de fecundidad se compararon mediante un ANOVA usando Minitab para Windows (versión 16; Minitab Inc., State College, PA). Las medias con diferencias significativas fueron comparadas usando el método de Tukey. La tasa sexual fue transformada mediante arco seno raíz cuadrada, antes del análisis estadístico.

\section{RESULTADOS}

En la comparación del desempeño mediante parámetros poblacionales (Cuadro 1) se encontraron para Ro, valores que varían desde 12.52 para la línea pura 2, hasta la mayor tasa, 86.24 para la población GV2. El tiempo generacional (T) en días, varía desde 13.8 para la línea pura 43 hasta 15.2 para la línea GV40. La tasa finita de crecimiento fue mayor a uno $(\lambda>1)$ para todas las líneas, prediciendo un incremento sostenido en todas las poblaciones. Por ultimo rm se manifiesta entre 0.178 para la línea pura 2 y 0.327 para la GV2 (Cuadro 1). 
Guzmán-Larralde et al.: Lineas puras y genéticamente variables de Trichogramma

Cuadro 1. Comparación de los parámetros poblacionales y fecundidad ( $\pm \mathrm{EE}$ ) de cuatro poblaciones de T. pretiosum: poblaciones compuestas por la combinación de 26 líneas puras con distinto número de generaciones (GV2 y GV40), y las líneas puras individuales 43 y 2.

\begin{tabular}{ccccccccc}
\hline \multicolumn{3}{c}{ Parámetros poblacionales } & \multicolumn{4}{c}{ Fecundidad } \\
\hline & Ro & $\mathrm{T}$ & $\lambda$ & $\mathrm{r}_{\mathrm{m}}$ & $\begin{array}{c}48 \mathrm{~h} \\
\text { promedio }\end{array}$ & & $\begin{array}{c}\text { total } \\
\text { promedio }\end{array}$ & \\
\hline GV2 & 86.24 & 14.232 & 1.386 & 0.327 & $35 \pm 1.64$ & $\mathrm{~A}$ & $215 \pm 8.80$ & $\mathrm{~A}$ \\
GV40 & 82.651 & 15.193 & 1.368 & 0.313 & $30 \pm 1.28$ & $\mathrm{~A}$ & $179 \pm 10.70$ & $\mathrm{~B}$ \\
43 & 56.935 & 13.761 & 1.355 & 0.304 & $32 \pm 1.63$ & $\mathrm{~A}$ & $111 \pm 7.85$ & $\mathrm{C}$ \\
2 & 12.524 & 15.181 & 1.195 & 0.178 & $19 \pm 2.21$ & $\mathrm{~B}$ & $54 \pm 7.39$ & $\mathrm{D}$ \\
\hline
\end{tabular}

GV2, población genéticamente variable con dos generaciones; GV40, población genéticamente variable con 40 generaciones; 43 , línea pura; 2 , línea pura; Ro, tasa neta reproductiva; $T$, tiempo medio generacional; $\lambda$ tasa finita de crecimiento; rm, tasa intrínseca de crecimiento. Promedios seguidos por la misma letra no son diferentes significativamente. Tukey $(\mathrm{P}<0.001)$. Temperatura $25^{\circ} \mathrm{C}, 16 / 8$-h luz/obscuridad y $30 \%$ humedad relativa.

Además se encontraron diferencias significativas en el análisis de fecundidad a las $48 \mathrm{~h}\left(F_{3,82}=13.50 ; P<0.001\right)$ y en la fecundidad total $\left(F_{3,82}=48.60 ; P<0.001\right)$. El análisis de medias muestra dos grupos bien definidos para la fecundidad a las $48 \mathrm{~h}$. Uno compuesto por la línea pura 2, que fue la que presentó los valores de fecundidad más bajos $(19 \pm 2.21)$ y otro compuesto por las tres poblaciones restantes; siendo la GV2 la que presentó los valores más altos de fecundidad (35 \pm 1.64$)$. Para la fecundidad total las medias muestran diferencias significativas entre cada una de las cuatro poblaciones comparadas, siendo la GV2 la que presentó los valores más altos (215 \pm 8.8). (Cuadro 1).

En las comparaciones de la tasa sexual se encontraron diferencias significativas en el análisis de varianza de las $48 \mathrm{~h}\left(F_{3,82}=15.04 ; P<0.001\right)$ y en los datos totales $\left(F_{3,82}=5.79 ; P=0.001\right)$. La comparación de medias a las $48 \mathrm{~h}$ muestra dos grupos bien definidos, uno con la proporción de macho más alta compuesto por la línea 2 y otro grupo por las tres poblaciones restantes; siendo la GV2 la que presentó una menor proporción de machos $(0.14 \pm 0.050)$. Así mismo los datos totales de la tasa sexual se forman dos grupos, siendo las que presentan una menor proporción de machos las líneas 2 y $43(0.52 \pm 0.001$ y $0.48 \pm 0.001$ respectivamente $)$ y la que tiene una mayor proporción de machos GV2 $(0.78 \pm 0.001)$ (Cuadro 2).

\section{DISCUSIÓN}

En la comparación del desempeño mediante los parámetros poblacionales, podemos observar diferencias entre las poblaciones genéticamente variables y las líneas puras (Cuadro 1). La combinación de un mayor número de líneas con menos generaciones, constituye la población con mejor desempeño. Esto concuerda con estudios hechos 
Cuadro 2. Resultados de las comparaciones de la tasa sexual (machos) a las $48 \mathrm{hrs}$ y datos totales.

\begin{tabular}{cccccc}
\hline & \multicolumn{4}{c}{ Proporción sexual } \\
\hline Poblaciones & N & \% machos & grupos & $\%$ machos & grupos \\
\hline 2 & 12 & $0.45 \pm 0.108$ & B & $0.52 \pm 0.001$ & A \\
43 & 25 & $0.18 \pm 0.103$ & A & $0.48 \pm 0.001$ & A \\
GV40 & 24 & $0.17 \pm 0.038$ & A & $0.62 \pm 0.002$ & A B \\
GV2 & 25 & $0.14 \pm 0.050$ & A & $0.78 \pm 0.001$ & B \\
\hline
\end{tabular}

GV2, Población genéticamente variable con dos generaciones; GV40, Población genéticamente variable con 40 generaciones; 43, línea pura; 2, línea pura. Promedios seguidos por la misma letra no son diferentes significativamente. Tukey $(\mathrm{P}<0.001)$. Temperatura $25^{\circ} \mathrm{C}, 16 / 8$-h luz/obscuridad y $30 \%$ humedad relativa.

dos años antes por (Guzmán-Larralde et al. 2013), en un hospedero distinto, donde comparó poblaciones genéticamente variables con distinto número de generaciones, líneas puras y de laboratorios de reproducción masiva. Sin embargo es importante destacar que la fecundidad a las 48 horas no presenta diferencias entre las dos poblaciones genéticamente variables GV2 y GV40 y la línea 43, información que toma relevancia al valorar que en la naturaleza los individuos tienen una mayor presión de selección del ambiente, y por lo tanto, un menor tiempo de sobrevivencia comparada con la que se presenta en el laboratorio. Una alta fecundidad durante los primeros días concuerda con estudios en Trichogramma minutum Riley y Trichogramma chelonis Ishii cuando se provee un ilimitado número de huevecillos diariamente (Bai \& Smith 1993, Miura \& Kobayashi 1995). Además se observó una reducción de las tasas poblacionales y de la fecundidad total de la línea genéticamente variable GV40, al incrementar el número de generaciones, lo que sugiere una reducción del desempeño de la población a través del tiempo. Esta información coincide con los estudios hechos por (Woodworth et al. 2002) en donde encontró un deterioro genético y una reducción en el desempeño en poblaciones de Drosophila después de 50 generaciones en condiciones de laboratorio. Por lo tanto, se quiere reducir el costo de la renovación de la variabilidad genética de la población en cautiverio, una alternativa sería utilizar una línea pura con alto desempeño en laboratorio y campo.

Lo más deseable en una reproducción de parasitoides es un mayor número de hembras, ya que estas influyen directamente en la reducción del hospedero. La proporción de sexos a las $48 \mathrm{~h}$, señala a las poblaciones genéticamente variables GV2 y GV40 y la línea pura 43, como aquellas que producen un mayor número de hembras. Sin embargo esta relación cambia radicalmente en el balance total en el caso de las dos líneas genéticamente variables, pero no así en la línea 43, la cual mantiene su condición basada en hembras $(0.48 \pm 0.001)$. La proporción sexual encontrada en hembras de esta misma línea 43 en un experimento realizado en Febrero 2011 (Guzmán-Larralde 
et al. 2013), y la encontrada en este estudio (Mayo 2013), con más de 2 años entre ellos y aproximadamente 95 generaciones, se mantuvo constante, lo que le confiere ventajas al uso de líneas puras, esto concuerda con la investigación de (Antolin 1999) la cual recapitula distintos trabajos con especies puras haplodiploides, incluyendo a T. pretiosum, y en donde no encuentra cambios en la proporción sexual.

Es indudable que aún falta investigación acerca del desempeño en campo, usando líneas puras exitosas en estudios de laboratorio. En resumen, subdividir una población de campo en líneas puras asegura la permanencia de ciertas características (alelos) en el tiempo y combinar las mejores líneas dependiendo del desempeño específico a condiciones climáticas y biológicas, previo a su liberación, promueve ventajas en los resultados que los centros de reproducción masiva buscan, incrementando y manteniendo las características deseadas a través de innumerables generaciones.

AGRADECIMIENTOS. Un agradecimiento especial al CONACyT, UCR y UAAAN por su apoyo económico para la realización del presente estudio.

\section{LITERATURA CITADA}

Allendorf, F.W. 1986. Genetic drift and the loss of alleles versus heterozygosity. Zoo Biology, 5: 181190.

Antolin, M. F. 1999. A genetic perspective on mating systems and sex ratios of parasitoid wasps. Researches on Population Ecology, 41: 29-37.

Arredondo Bernal, H. C. 2010. Directorio de Laboratorios Reproductores y comercializadores de Agentes de Control Biológico.

Badii M. H. \& Castillo, J. 2009. Muestreo Estadístico: Conceptos y Aplicaciones. UANL.

Bai, B. \& Smith, S. M. 1993. Effect of host availability on reproduction and survival of the parasitoid wasp Trichogramma minutum. Ecological Entomology, 18: 279-286.

Bartlett, A. C. 1984. Genetic changes during insect domestication. Pp. 2-8. In: E. G. King \& N. C. Leppla (Eds.). Advances and Challenges in Insect Rearing. U.S. Department of Agriculture Handbook, USDA, New Orleans, LA.

Bellows Jr, T. S., Van Driesche, R. G. \& Elkinton, J. S. 1992. Life-table construction and analysis in the evaluation of natural enemies. Annual Review of Entomology, 37: 587-612.

Cerutti F. \& Bigler, F. 1995. Quality assessment of Trichogramma brassicae in the laboratory. Entomologia Experimentalis et Applicata, 75: 19-26.

Chassain C. \& Boulétreau, M. 1991. Genetic variability in quantitative traits of host exploitation in Trichogramma (Hymenoptera: Trichogrammatidae). Genetica, 83: 195-202.

Frankham, R. 2005. Genetics and extinction. Biological Conservation, 126: 131-140.

Guzmán-Larralde, A., Cerna-Chávez, E., Rodríguez-Campos, E., Loyola-Licea, J. C. \& Stouthamer, R. 2013. Genetic variation and the performance of a mass-reared parasitoid, Trichogramma pretiosum (Hymenoptera: Trichogrammatidae) in laboratory trials. Journal of Applied Entomology, DOI: $10.1111 /$ jen. 12073 .

Haile, A. T., Hassan, S. A., Sithanantham, S., Ogol, C. K. P. O. \& Baumgärtner, J. 2002. Comparative life table analysis of Trichogramma bournieri Pintureau and Babault and Trichogramma sp. nr. mwanzai Schulten and Feijen (Hym., Trichogrammatidae) from Kenya. Journal of Applied Entomology, 126: 287-292. 
Hopper, K.R., Roush, R. T. \& Powell, W. 1993. Management of genetics of biological-control introductions. Annual Review of Entomology, 38: 27-51.

Hufbauer, R. A. \& Roderick, G. K. 2005. Microevolution in biological control: mechanisms, patterns, and processes. Biological Control, 35: 227-239.

Iranipour, S., Farazmand, A., Saber, M. \& Mashhadi, J. 2009. Demography and life history of the egg parasitoid, Trichogramma brassicae, on two moths Anagasta kuehniella and Plodia interpunctella in the laboratory. Journal of Insect Science, 9: 1-8.

Kalyebi, A., Overholt, W. A., Schulthess, F., Mueke, J. M., Hassan, S. A. \& Sithanantham, S. 2005. Functional response of six indigenous trichogrammatid egg parasitoids (Hymenoptera: Trichogrammatidae) in Kenya: influence of temperature and relative humidity. Biological Control, 32: 164-171.

Kazmer, D. J. \& Luck, R. F. 1995. Field tests of the size-fitness hypothesis in the egg parasitoid Trichogramma pretiosum. Ecology, 76: 412-425.

Leslie, P. H. 1945. On the Use of Matrices in Certain Population Mathematics. Biometrika, 33: 183212.

Lewis, E. G. 1942. On the Generation and Growth of a Population. Sankhyā: The Indian Journal of Statistics, (1933-1960) 6: 93-96.

Li, C. C. 1955. The Stability of an Equilibrium and the Average Fitness of a Population. The American Naturalist, 89: 281-295.

Lotka, A. J. 1907. Studies on the mode of growth of material aggregates. American Journal of Science, 24: 199-216.

Maia, A. H. N., Luiz, A. J. B. \& Campanhola, C. 2000. Statistical inference on associated fertility life parameters using jackknife technique: computational aspects. Journal of Economic Entomology, 93: 511-518.

Miura, K. \& Kobayashi, M. 1995. Reproductive properties of Trichogramma chilonis females on diamondback moth eggs. Applied Entomology and Zoology, 30: 393-393.

Nunney, L. 2003. Managing captive populations for release: a population genetic perspective, pp. 7388. In: van Lenteren (Ed.). Quality Control and production of Biological Control Agents: Theory and Testing Procedures. CABI publishing, Wallingford, UK.

Nunney, L. 2006. Captive Rearing for Field Release: A Population Genetic Perspective, pp. 1-4. In: D. Pimentel (Ed.). Encyclopedia of Pest Management. Taylor \& Francis, London.

Nunney, L., Leppla, N. C., Bloem, K. A. \& Luck, R. F. 2002. The population genetics of mass-rearing. Pp. 43-49. In: N.C. Leppla, K.A. Bloem \& R.F. Luck (Eds.). Quality control for mass-reared arthropods Proceedings of the eighth and ninth workshops of the IOBC working group on quality control of mass-reared arthropods. 9-12 October 1995, Santa Barbara, CA and 2-4 March 1998, Cali, Colombia.

Pak, G.A. \& Oatman, E. R. 1982. Comparative life table, behavior and competition studies of Trichogramma brevicapillum and T. pretiosum. Entomologia experimentalis et applicata, 32: 68-79.

Pinto, J. D. 1998. Systematics of the North American species of Trichogramma Westwood (Hymenoptera: Trichogrammatidae). Memoirs of the Entomological Society of Washington, 22: 1-287.

Pratissoli, D. \& Parra, J. R. P. 2000. Fertility life table of Trichogramma pretiosum (Hym., Trichogrammatidae) in eggs of Tuta absoluta and Phthorimaea operculella (Lep., Gelechiidae) at different temperatures. Journal of Applied Entomology, 124: 339-342.

Pratissoli, D., Zanuncio, J. C., Vianna, U. R., Andrade, J. S., Guimarães, E. M. \& Espindula, M. C. 2004. Fertility life table of Trichogramma pretiosum and Trichogramma acacioi on eggs of Anagasta kuehniella at different temperatures. Pesquisa Agropecuária Brasileira, 39: 193-196. 
Roush, R. T. \& Hopper, K. R. 1995. Use of single family lines to preserve genetic variation in laboratory colonies. Annals of the Entomological Society of America, 88: 713-717.

Samara, R.Y., Carlos Monje, J. \& Zebitz, C. P. W. 2008. Comparison of different European strains of Trichogramma aurosum (Hymenoptera: Trichogrammatidae) using fertility life tables. Biocontrol Science and Technology, 18: 75-86.

Sorati, M., Newman, M. \& Hoffmann, A. A. 1996. Inbreeding and incompatibility in Trichogramma $n r$. brassicae: evidence and implications for quality control. Entomologia experimentalis et applicata, 78: 283-290.

Southwood, T. R. E. 1978. Ecological methods, with particular reference to the study ofinsect populations. Chapman and Hall London.

Van Lenteren, J. C., Drost, Y. C., van Roermund, H. J. W. \& Posthuma-Doodeman, C. J. a. M. 1997. Aphelinid parasitoids as sustainable biological control agents in greenhouses. Journal of Applied Entomology, 121: 473-485.

Waage, J. K. \& Ming, N. S. 1984. The Reproductive Strategy of a Parasitic Wasp: I. Optimal Progeny and Sex Allocation in Trichogramma evanescens. Journal of Animal Ecology, 53: 401-415.

Wajnberg, E. 2004. Measuring Genetic Variation in Natural Enemies Used for Biological Control: Why and How? Pp. 19-37. In: L.E. Ehler, R. Storza \& T. Mateille (Eds). Genetics, evolution and biological control. CABI Publishing, Wallingford, UK.

Wilson, K. \& Hardy, I. C. W. 2002. Statistical analysis of sex ratios: an introduction. Pp. 48-92. In: I.C.W. Hardy (Ed). Sex Ratios: Concepts and Research Methods. Cambridge University Press.

Woodworth, L. M., Montgomery, M. E., Briscoe, D. A. \& Frankham, R. 2002. Rapid genetic deterioration in captive populations: Causes and conservation implications. Conservation Genetics, 3: 277-288. 